\title{
Chemically/Magnetically Dual-Responsive Nanoparticles for Multipurpose Colorimetric Sensor
}

\author{
Wei Liu, ${ }^{*}$ Xuefeng Liu, ${ }^{1}$ Jiabao Ren, ${ }^{1}$ Chen Cui, ${ }^{1}$ and Shujie $\mathrm{Xu}{ }^{1}$ \\ ${ }^{1}$ Automotive Material Research Department, Automotive Data of China Co., Ltd., Tianjin, 300300, China
}

\begin{abstract}
Magnetically responsive colloidal photonic crystals can change their structural color according to the external magnetic field, which has been widely studied in recent years. However, due to lack of recognition ability towards non-magnetic analytes, these photonic crystals can be applied to constructing a sensor only when an additional stimuli responsive unit is involved. To address this problem, we used a functional protein to modify the magnetically responsive colloidal particles to construct chemically/magnetically dualresponsive nanoparticles. For a proof of concept research in this manuscript, we modified the colloidal particles with streptavidin, and the as obtained nanoparticles were used to detect biotinylated protein via a binding and assembling strategy, which is impossible for conventional photonic crystal sensors. Not only qualitative and quantitative detections were achieved, but also the average diameters of the biotinylated protein were correctly estimated. These results have demonstrated a multipurpose detection feature of our proposed colorimetric sensor.
\end{abstract}

\section{Introduction}

Upon application of an external magnetic field, magnetically responsive colloidal particles with diameters in the range of $c a$. 70 to $290 \mathrm{~nm}$ can assemble into photonic chains within seconds based on the balance of attractive (magnetic) and repulsive (electrostatic or steric) forces. ${ }^{1-7}$ When observed with an incident light, the obtained periodical structure reveals structural colors which could be interpreted by Bragg's law $(\lambda=2 n d \sin \theta$, where $\lambda$ is the diffraction wavelength, $n$ the refractive index of dispersant, $d$ the lattice constant, and $\theta$ the Bragg angle). ${ }^{8}$ The variables $d$ and $\theta$ can be manipulated magnetically by varying the magnetic field, or tuned non-magnetically by stimuli responsive polymers, to achieve desired structural colors. Based on this principle, many intriguing applications have been reported such as magnetically controlled display units, ${ }^{9-11}$ photonic anticounterfeiting, ${ }^{12,}{ }^{13}$ color encoding for bioassays, ${ }^{14}$ photonic humidity sensors, ${ }^{15,16}$ and rewritable photonic papers. ${ }^{17,18}$ Relative reviews have also been reported. ${ }^{19-21}$ Taking the humidity sensor as an example, magnetically responsive colloidal particles were magnetically assembled into photonic chains and then fixed in hygroscopic polymers. The polymer matrix could swell upon contact with humid air, leading to the increase of lattice constant $(d)$ and consequently brilliant color change from blue to red, which could be observed visually. Compared with other non-magnetic materials, the facile and fast magnetically induced assembly property of these colloidal particles is highly desirable to construct photonic crystal sensors. However, due to the lack of recognition ability towards non-magnetic analytes, the colloidal particles can be applied to constructing photonic crystal sensors only when an additional stimuli responsive unit such as the hygroscopic polymers mentioned above is involved. This drawback has significantly hindered the wide application of magnetically responsive colloidal particles in sensing areas.

On the other hand, photonic crystal sensors that could give visually perceptible color change according to the amount of analyte are of increasing interest in recent years because of their low-cost nature and simplicity. ${ }^{22-24}$ Highly ordered periodical structures of the photonic crystals made by materials such as $\mathrm{SiO}_{2}$ or polystyrene colloidal particles are fixed to form mesoporous Bragg stacks, ${ }^{25}$ hydrogel or polymer networks, ${ }^{26-29}$ or suspension arrays composed of encoded microparticles. ${ }^{30-32}$ Diffraction occurs when the periodicity of structures and the wavelength of incident light satisfies the Bragg condition. These structures are functionalized to respond to specific analytes to result in changes of their structural parameters such as size or refractive index, consequently the diffraction wavelengths, and eventually the structural colors. Though many researches have achieved structural colors response to several different biochemical analytes, their fabrication processes are always challenging and timeconsuming. Not only the highly ordered periodical structures should be maintained, but also the sensitivity and specificity against analytes should be provided during the processes.

*E-mail: liuwei201507@ catarc.ac.cn 
Here, for the first time, we report the development of a colorimetric sensor based on chemically/magnetically dual-responsive nanoparticles (CMDRP) and its use for multipurpose detection of biomolecules. Not only concentrations of the analytes were qualitatively and quantitatively detected, but also average diameters of the analytes were correctly estimated based on the Bragg's law. The chemically responsive ability of the CMDRP was achieved by directly modifying the magnetically responsive colloidal particles with a functional protein. When an analyte was presented, the average diameter of the CMDRP increased through protein-analyte interaction. As a result, the lattice constant $(d)$ of the magnetically induced photonic chains also increased, and a red shift of the diffraction wavelength was achieved. As proteins with different biorecognition abilities always have similar functional groups (primary amine in this case), it is convenient to fabricate CMDRP based colorimetric sensors for various analytes without modifying the fabrication process. In addition, the magnetically responsive property of the obtained CMDRP made it possible to form the periodical structure after an analyte being recognized by the functional protein. We call this as a binding and assembling strategy. That is to say, the indispensable structure-fixing procedure for the fabrication of conventional photonic crystal sensors is not required for our proposed strategy. Therefore the fabrication complexity of the colorimetric sensor is significantly reduced. We believe that this novel CMDRP based binding and assembling detection paradigm can easily fabricate colorimetric sensors for a variety of analytes.

\section{Experimental section}

\subsection{Fe $\mathrm{O}_{4} @ \mathrm{C} @ S A$ preparation}

All chemicals used in this study were of analytical grade and used as received without further purification. Synthesis of carbon-encapsulated superparamagnetic colloidal particles was carried out following the literature procedure.33 A stainless steel-lined autoclave was used instead of a Teflon-lined autoclave. Hydrogen peroxide (30\%) of $1.2 \mathrm{ml}$ and $1.5 \mathrm{ml}$ were used as the oxidant to synthesize the particles with average diameters of 170 $\mathrm{nm}$ and $213 \mathrm{~nm}$, respectively. A typical two-step procedure for EDC/NHS crosslinking of the carboxyl groups on $\mathrm{Fe} 3 \mathrm{O} 4 @ \mathrm{C}$ with primary amines on protein was used. The obtained Fe3O4@C particles in acetone were washed and dispersed in DI water by ultrasonic dispersion, followed by repeated washing using MES buffer (0.1 M, pH6.0) and magnetic separation. After resuspending and mixing the particles in MES buffer containing 0.4 M EDC and 0.1 M Sulfo-NHS for $20 \mathrm{~min}$, MES buffer washing was applied again to removing excess EDC and Sulfo-NHS. PBS buffer (0.05 M, pH7.4, ionic strength of $0.15 \mathrm{M}$ adjusted by $\mathrm{NaCl}$ ) was used to resuspend the particles, then streptavidin (SA, SigmaAldrich, at the ratio of $0.06 \mathrm{mg} \mathrm{SA}$ to $1 \mathrm{mg}$ magnetic particles) was added to the solution and left to react for 2 $\mathrm{h}$ at room temperature under mixing. Then the mixture was adjusted to $\mathrm{pH} 8.5$ by adding $\mathrm{NaOH}$, and left to react for $30 \mathrm{~min}$ to regenerate the unreacted carboxyl groups. PBS buffer containing $1 \%$ BSA was then used to suspend and wash the SA functionalized particles. The obtained Fe3O4@C@SA was finally stored at $4^{\circ} \mathrm{C}$ in DI water. The analytes biotinylated BSA (b-BSA) and biotinylated $\operatorname{IgG}$ (b-IgG) were purchased from Thermo Scientific and Sigma-Aldrich, respectively.

\subsection{Characterizations}

Fourier-transform infrared spectra were recorded using a Nicolet Nexus 470 FT-IR spectrophotometer, within the range from 400 to $4000 \mathrm{~cm}-1$. X-ray photoelectron spectroscopy (XPS) data were obtained using a PHI 5000 VersaProbe (Physical Electronics, Inc.), equipped with a monochromatized aluminum X-ray source. For scanning electron microscope (SEM), the samples were sputtercoated with platinum using an E1045 Pt-coater (Hitachi High-technologies CO.) and then imaged by an S-4800 field emission scanning electron microscope (Hitachi High-technologies CO.) at an acceleration voltage of 3.0 $\mathrm{kV}$. ImageJ software was used to process the SEM images following the procedures of find edges, east shadows, and invert LUT. The particle size distributions were measured with Zetasizer Nano ZS (Malvern Instruments Ltd.). The reflection spectra were recorded using a fiber spectrometer (HR2000+, Ocean Optics) with an integration time of $500 \mathrm{~ms}$. A cuvette with optical length of $2 \mathrm{~mm}$ was placed between the fiber probe and a permanent magnet $(\mathrm{NdFeB}, 12.7 \mathrm{~mm} \times 25.4$ $\mathrm{mm} \times 50.8 \mathrm{~mm}$ ), and used to hold the sample suspensions for reflection spectra measurements. WS-1 diffuse reflectance standard (Ocean Optics) covered by a piece of cuvette glass was used as reference standard. The measurement angle between Y-shaped fiber probe (QR400-7-VIS-NIR, Ocean Optics) and the cuvette normal was $0^{\circ}$, while the distance between the probe and the cuvette was ca. $0.2 \mathrm{~mm}$.

\section{Results and discussion}

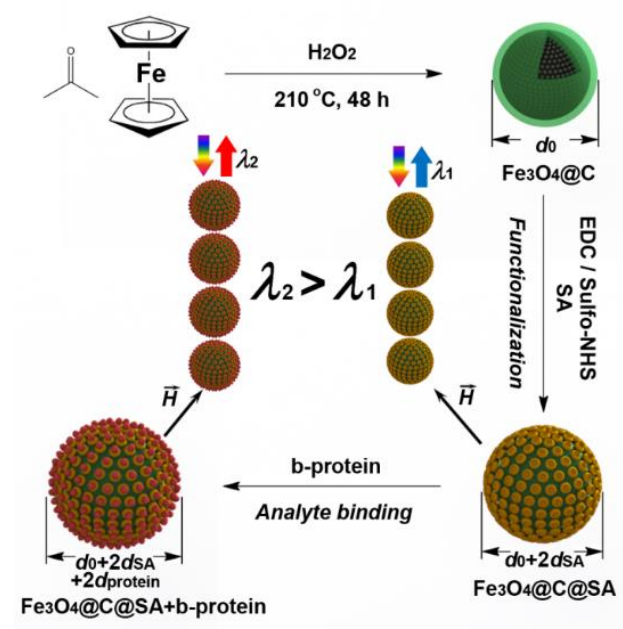

Fig. 1 General fabrication process and detection basis of the CMDRP based colorimetric sensor 
To synthesize a CMDRP, the magnetically responsive colloidal particles should have proper functional groups on their surfaces to carry out covalent coupling with the functional protein. There are several candidates of which the surfaces have suitable decorations such as polyacrylic acid,1 $\mathrm{SiO} 2,34$ or carbon.35 For a proof of concept research, we chose carboxyl-contained carbonencapsulated particles (Fe3O4@C) as the building blocks to fabricate the CMDRP based colorimetric sensor. General fabrication process and detection basis of the proposed binding and assembling detection strategy were shown in Fig. 1. SA was selected as the functional protein, b-BSA and b-IgG were selected as the analytes, which took advantage of the biotin-avidin model system in establishing a biosensing platform. The carboxyl groups outside the Fe3O4@C were covalently coupled with SA by a typical EDC/NHS chemistry. Successful SA functionalization of the $\mathrm{Fe} 3 \mathrm{O} 4 @ \mathrm{C}$ was confirmed by FT-IR and XPS spectra as shown in Fig. 2. For the FT-IR spectra, bands belongings are as follows, 1702: $\mathrm{C}=\mathrm{O}$ of carboxyl groups; 1655: $\mathrm{C}=\mathrm{O}$ of amide bond; 1597: conjugated double bond of cyclopentadienyl groups; 1530: N-H of amide bond; 584: Fe3O4. These data indicated that the obtained Fe3O4@C@SA CMDRP possessed combined characteristics belonged to SA and Fe3O4@C. For the wide scan XPS spectra, relative atomic ratio of nitrogen of SA, Fe3O4@C, and Fe3O4@C@SA is $13.0 \%, 0 \%$, and 2.72\%, respectively, which demonstrated the appearance of protein on the surface of Fe3O4@C particles. For the high-resolution C1s XPS spectra, the peak at $289.0 \mathrm{eV}$ accounting for carbon atoms in the carboxyl groups demonstrated the capability of $\mathrm{Fe} 3 \mathrm{O} 4 @ \mathrm{C}$ being functionalized via EDC/NHS chemistry. The peak at $285.9 \mathrm{eV}$ accounting for carbon atoms in the $\mathrm{C}-\mathrm{N}$ groups also demonstrated the appearance of protein on the surface of $\mathrm{Fe} 3 \mathrm{O} 4 @ \mathrm{C}$ particles.
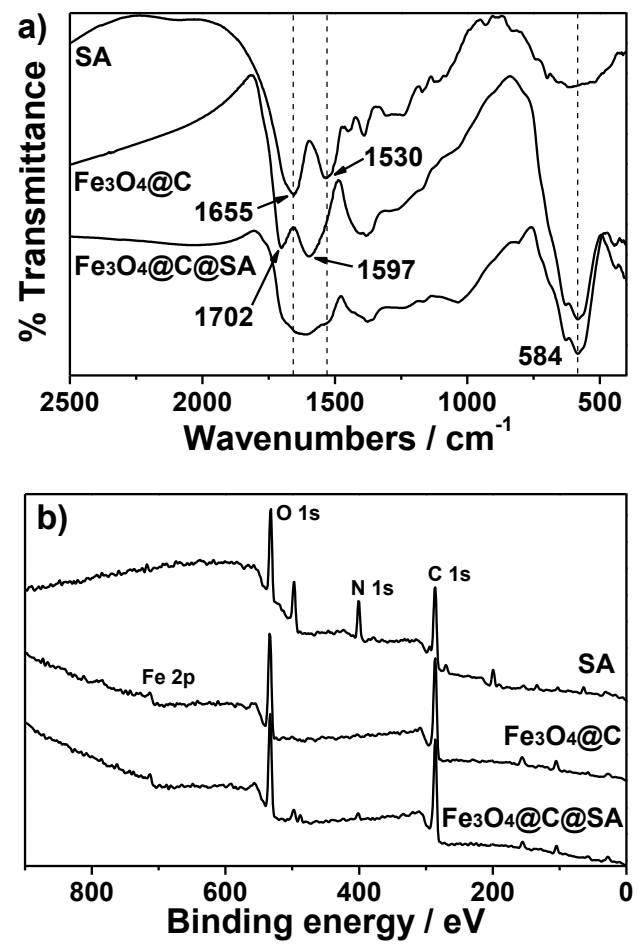

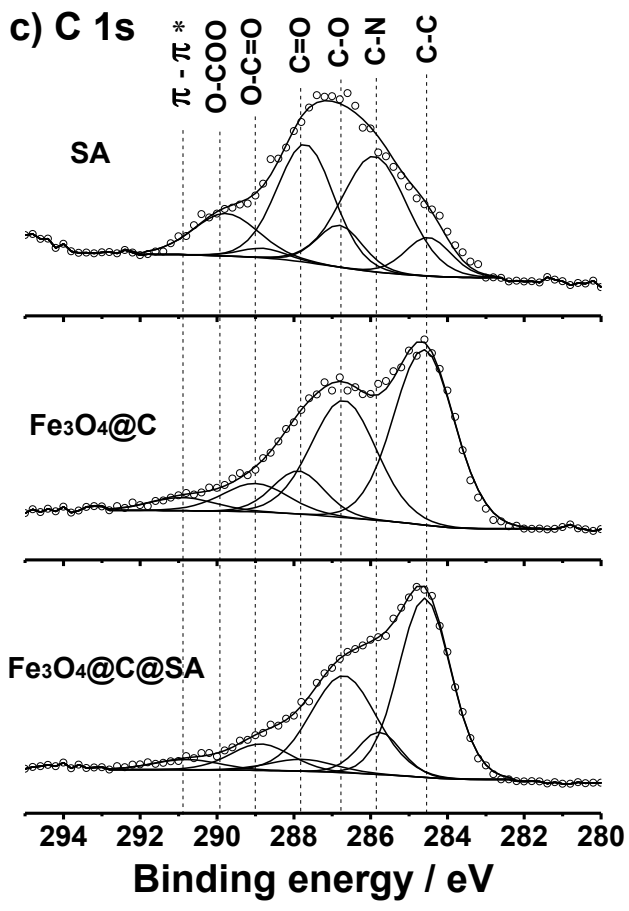

Fig. 2 a) FT-IR spectra, b) wide scan XPS spectra, and c) highresolution C1s XPS spectra of the samples.

The as obtained ready-to-use Fe3O4@C@SA suspended in deionized (DI) water was then used to qualitatively detect b-BSA with the binding and assembling detection strategy. The Fe3O4@C@SA (3.4 mg ml-1) was incubated with excess b-BSA in DI water under mixing for $1 \mathrm{~h}$. As a result of the binding between SA and b-BSA, the particle diameter was increased. After magnetic separation (magnetic field of $0.16 \mathrm{~T}$, field gradient of $0.10 \mathrm{~T} \mathrm{~cm}-1,10 \mathrm{~min}$ ) and resuspension in DI water by ultrasonic dispersion, obvious red shift of the structural color was achieved upon application of an external magnetic field. Fig. 3a showed the particle size distributions measured by dynamic light scattering (DLS) and particle morphologies obtained by SEM. Hydrodynamic diameters of the nanoparticles were increased as the protein layers thickened $(213 \pm 10.9 \mathrm{~nm}$, $216 \pm 8.74 \mathrm{~nm}$, and $237 \pm 12.0 \mathrm{~nm}$ for $\mathrm{Fe} 3 \mathrm{O} 4 @ \mathrm{C}$, Fe3O4@C@SA, and Fe3O4@C@SA+b-BSA, respectively). Careful inspection revealed that the roughness of the particle surfaces was also increased due to protein decoration. These data further demonstrated that the Fe3O4@C was successfully functionalized by SA, and the as obtained Fe3O4@C@SA still possessed biotin-binding activity. Fig. 3b showed that as the SA functionalization and b-BSA binding proceeded, the diffraction wavelengths of the nanoparticles gradually shifted red. These color shifts directly demonstrated the chemically/magnetically dual-responsive feature of the obtained Fe3O4@C@SA. Therefore, the analyte b-BSA could be detected qualitatively and, more importantly, be visually observed by the proposed CMDRP based colorimetric sensor via a simple, low-cost, and label-free manner. 

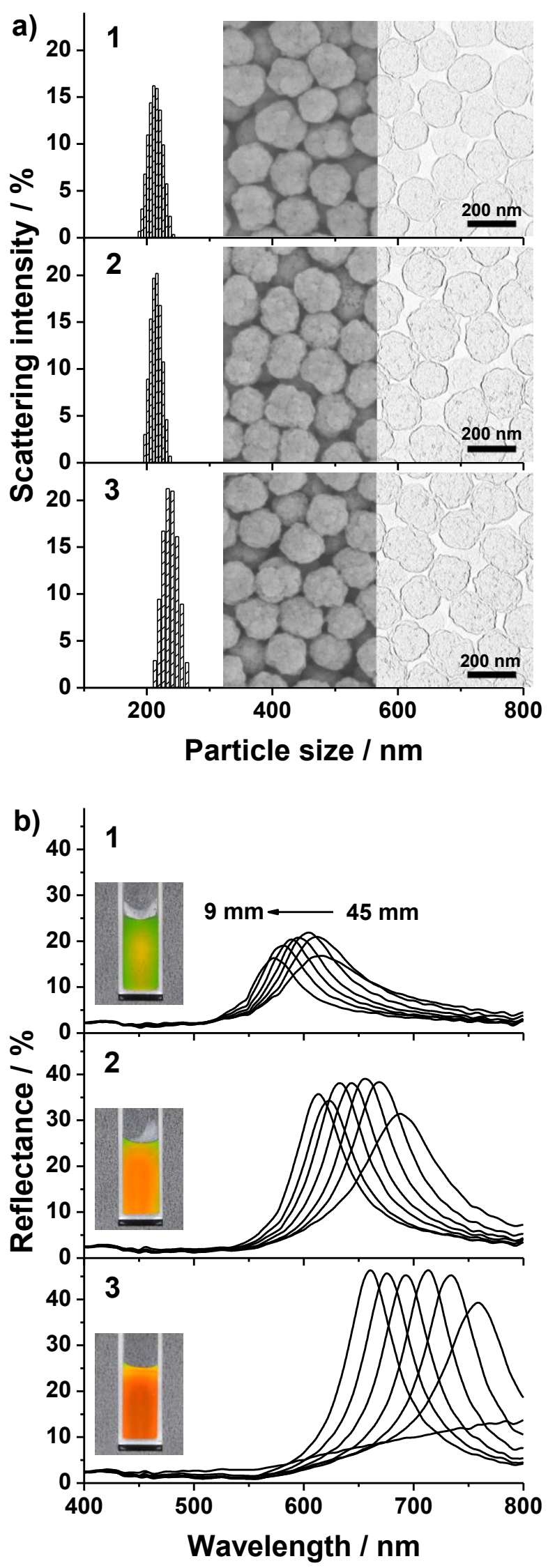

Fig. 3 a) Particle size distributions measured by DLS and corresponding SEM images of 1: $\mathrm{Fe}_{3} \mathrm{O}_{4} @ \mathrm{C}, 2: \mathrm{Fe}_{3} \mathrm{O}_{4} @ \mathrm{C} @ \mathrm{SA}$, and 3: $\mathrm{Fe}_{3} \mathrm{O}_{4} @ \mathrm{C} @ \mathrm{SA}+\mathrm{b}-\mathrm{BSA}$. Half of the images were processed by ImageJ software to facilitate observation of the sample surfaces. b) Reflection spectra under different magnetic fields and typical photographs of the nanoparticles suspended in DI water. The diffraction wavelengths shifted blue as the magnet-sample distance decreased from $45 \mathrm{~mm}$ to $9 \mathrm{~mm}$ with step size of $6 \mathrm{~mm}$; the photographs were captured with the distance of $9 \mathrm{~mm}$.

Formation of magnetically induced photonic chains with different lattice constants (d) is the basis of CMDRP based colorimetric sensor, which can be used to estimate the protein size based on the Bragg's law $\left(\theta=90^{\circ}\right.$ and $n$ $=1.33$ for water in this study). While similar principle has been applied to estimating the thickness of solvation layer of magnetically responsive nanoparticles suspended in various solvents, 34, 36 such a protein-sizedetermination capability of our strategy has, so far as we known, not been reported. The magnetically induced dipole-dipole attraction between Fe3O4@C particles is balanced with three interparticle repulsion layers including incompressible solvation layer, incompressible protein layer, and compressible electrostatic layer.3, 34, 36 Fig. 4a illustrated two typical photonic chain situations of Fe3O4@C@SA with external magnetic fields $\mathrm{H} 2$ bigger than $\mathrm{H} 1$. As all the detection manipulations were conducted in DI water, the influence of solvation layer was not considered in our strategy. This approximation was compensated by using the hydrodynamic size measured by DLS $(213 \pm 10.9 \mathrm{~nm})$ instead of the size measured by SEM $(199 \pm 18.7 \mathrm{~nm}$ by measuring 150 particles) as the diameter of Fe3O4@C. Therefore, the Bragg's law was expressed as that shown in Fig. 4a, in which the average diameter of protein could be estimated by processing the data of Fig. 3b, and the results were shown in Fig. 4b.

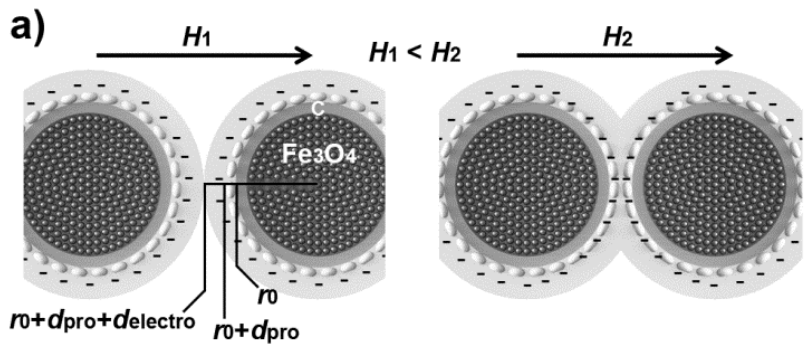

$$
\lambda=2 n(2 r o+2 d \text { pro }+2 d \text { electro })
$$

ro: hydrodynamic radius of $\mathrm{Fe}_{3} \mathrm{O} 4 @ \mathrm{C}$ dpro: average diameter of protein delectro: thickness of electrostatic repulsion layer

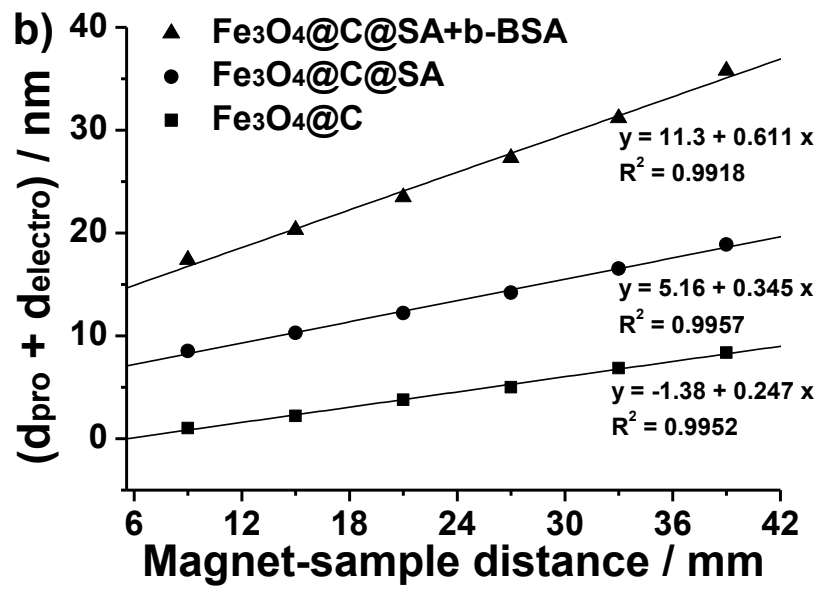


Fig. 4 a) Schematic illustration of the interparticle repulsion layers on the surface of $\mathrm{Fe}_{3} \mathrm{O}_{4} @ \mathrm{C} @$ SA. While the electrostatic repulsion layer is compressible when increasing the applied external magnetic field, the rigid protein layer is free from the influence of the magnetic field, thus the average diameter of protein can be estimated using the Bragg equation. $b$ )

Dependence of the $\left(d_{\text {pro }}+d_{\text {electro }}\right)$ thickness on the magnetsample distance. Data were obtained by processing the data of Fig. 3b.

As there is no protein layer on the surface of $\mathrm{Fe} 3 \mathrm{O} 4 @ \mathrm{C}$, its sole repulsive force comes from the compressible electrostatic repulsion layer. Based on the observed linear relationship between delectro of Fe3O4@C and the magnet-sample distance, a theoretical distance (x-intercept) that is capable of totally compressing the electrostatic repulsion layer can be obtained by extrapolating the fitted line. By calculating the fitted lines of $\mathrm{Fe} 3 \mathrm{O} 4 @ \mathrm{C} @ \mathrm{SA}$ and Fe3O4@C@SA+b-BSA with the obtained x-intercept, the average diameters of SA and BSA were estimated to be $7.09 \mathrm{~nm}$ and $7.62 \mathrm{~nm}$, respectively, which corresponded well with the actual sizes of these proteins (ca. $5.8 \times 8.4 \times 4.6 \mathrm{~nm}$ for SA and $4.5 \times 14.3 \times 10.9 \mathrm{~nm}$ for BSA, referenced from RCSB Protein Data Bank). Bearing in mind the size data obtained via DLS measurement as shown in Fig. 3a (ca. $1.5 \mathrm{~nm}$ for SA and $10.5 \mathrm{~nm}$ for BSA), our CMDRP based colorimetric sensor bioassay showed receivable accuracy in determination of protein sizes, also with a simple, lowcost, and label-free manner, which may find wide application in bioassay areas.

Besides qualitative detection of the presence of excess b-BSA and convincible determination of its average diameter, the proposed CMDRP based binding and assembling biosensing strategy can also detect protein analyte quantitatively. In this case, we chose bIgG as the analyte and Fe3O4@C with hydrodynamic diameter of $170 \pm 8.51 \mathrm{~nm}$ as the building blocks to construct the CMDRP based colorimetric sensor. Nanoparticles with smaller diameter were selected because shorter diffraction wavelength of the CMDRP could be achieved,5, 33 which contributed to easier naked-eye observations of detection results. When the

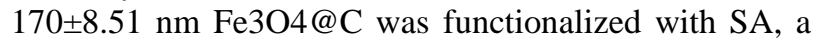
red shift from $460 \mathrm{~nm}$ to $552 \mathrm{~nm}$ of the diffraction wavelength was achieved with the magnet-sample distance of $9 \mathrm{~mm}$. We had also modified Fe3O4@C with hydrodynamic diameter of $148 \pm 6.57 \mathrm{~nm}$ with $\mathrm{SA}$, but no red shift was observed, mostly because of the lack of sufficient carboxyl groups outside the colloidal particles with such a small size. 33
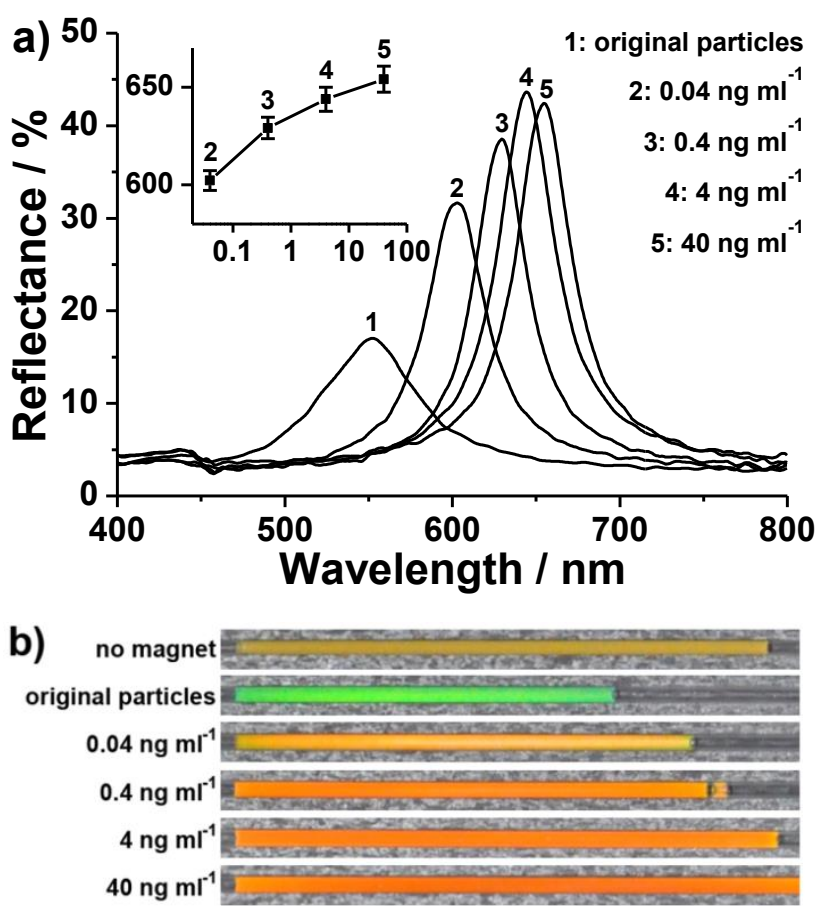

Fig. 5 a) Reflection spectra of the CMDRP based colorimetric sensor incubated with b-IgG with different concentrations. Inset: dependence of the diffraction wavelength on b- $\operatorname{IgG}$

concentration. The magnet-sample distance was $9 \mathrm{~mm}$; standard deviations of triplicate measurements were shown as error bars. b) Corresponding photographs of the nanoparticle suspensions held by capillaries ( $0.5 \mathrm{~mm}$ inner diameter) under the same magnetic field.

The quantitative detection was carried out by dissolving b-IgG in $4.5 \mathrm{ml}$ DI water with different concentrations, then $0.1 \mathrm{ml} \mathrm{Fe} 3 \mathrm{O} 4 @ \mathrm{C} @ \mathrm{SA}$ (8.8 mg ml1) was added and mixed at room temperature. After one hour incubation, samples were centrifuged $(2000 \times \mathrm{g}, 30$ min) and redispersed in $0.1 \mathrm{ml}$ DI water by ultrasonic dispersion. Reflectance spectroscopy was then applied to characterizing the final samples, and the results were shown in Fig. 5. The data showed that this un-optimized biosensing was capable of detecting b-IgG with an unexpected sensitivity. With the help of reflectance spectroscopy, a concentration range of $\mathrm{b}$ - $\mathrm{IgG}$ from 0.04 ng ml-1 to $40 \mathrm{ng} \mathrm{ml-1}$ was detectable. More importantly, the structural colors of the original particles, $0.04 \mathrm{ng} \mathrm{ml-}$ 1 experiment, and $0.4 \mathrm{ng} \mathrm{ml}-1$ experiment could be distinguished easily from each other when observed by naked-eye, which demonstrated the proposed CMDRP based binding and assembling detection as a simple yet powerful colorimetric sensing strategy. When the b-IgG concentration increased from $0.4 \mathrm{ng} \mathrm{ml}-1$ to $40 \mathrm{ng} \mathrm{ml-1}$, the corresponding diffraction wavelength shifted from $630 \mathrm{~nm}$ to $655 \mathrm{~nm}$, of which the colour changes were visually non-perceptible. This problem could be solved by optimizing the fabrication and operation parameters of the CMDRP based colorimetric sensor, such as the diameter of the Fe3O4@C building blocks and the magnetic field strength. Studies focusing on these issues are underway. 


\section{Conclusions}

In summary, chemically/magnetically dual-responsive nanoparticles (CMDRP), which could simultaneously respond to protein analyte and external magnetic field, were successfully fabricated and used to construct multipurpose colorimetric sensors. The most significant feature of the proposed CMDRP based colorimetric sensor is that the analyte recognition precedes the formation of the photonic crystal structure. We define this procedure as the binding and assembling strategy in this study, which is impossible for conventional photonic crystal sensors. Benefiting from the significant feature, qualitative detection of b-BSA and convincible determination of protein sizes of SA and BSA were accomplished via a simple, low-cost, and label-free manner. This feature, coupled with the magnetic separation, also made it possible to visually detect a small amount of b-IgG dissolved in a large amount of water without additional analyte concentration process. Based on the facile fabrication, simple operation, and multipurpose detection characteristics of the CMDRP colorimetric sensor, we believe that this novel material will have broad application in the area of colorimetric sensor.

\section{References}

1. J. Ge, Y. Hu and Y. Yin, Angew. Chem., 2007, 119, 7572-7575.

2. J. Ge, Y. Hu, M. Biasini, W. P. Beyermann and Y. Yin, Angew. Chem., Int. Ed., 2007, 46, 4342-4345.

3. W. Luo, H. Ma, F. Mou, M. Zhu, J. Yan and J. Guan, Adv. Mater., 2013, 26, 1058-1064.

4. H. Wang, Y. Sun, Q. Chen, Y. Yu and K. Cheng, Dalton T., 2010, 39, 9565-9569.

5. J. Ge, Y. Hu, T. Zhang, T. Huynh and Y. Yin, Langmuir, 2008, 24, 3671-3680.

6. J. Ge and Y. Yin, J. Mater. Chem., 2008, 18, 50415045.

7. L. He, V. Malik, M. Wang, Y. Hu, F. E. Anson and Y. Yin, Nanoscale, 2012, 4, 4438-4442.

8. L. He, M. Wang, J. Ge and Y. Yin, Account Chem. Res., 2012, 45, 1431-1440.

9. H. Hu, J. Tang, H. Zhong, Z. Xi, C. Chen and Q. Chen, Sci. Rep., 2013, 3.

10. J. Kim, Y. Song, L. He, H. Kim, H. Lee, W. Park, Y. Yin and S. Kwon, Small, 2011, 7, 1163-1168.

11. H. Kim, J. Ge, J. Kim, S. Choi, H. Lee, H. Lee, W. Park, Y. Yin and S. Kwon, Nat. Photon., 2009, 3, 534-540.

12. H. Hu, Q. Chen, J. Tang, X. Hu and X. Zhou, J. Mater. Chem., 2012, 22, 11048-11053.

13. R. Xuan and J. Ge, Langmuir, 2011, 27, 5694-5699.

14. H. Lee, J. Kim, H. Kim, J. Kim and S. Kwon, Nat. Mater., 2010, 9, 745-749.

15. H. Hu, Q. Chen, K. Cheng and J. Tang, J. Mater. Chem., 2012, 22, 1021-1027.
16. R. Xuan, Q. Wu, Y. Yin and J. Ge, J. Mater. Chem., 2011, 21, 3672-3676.

17. J. Ge, J. Goebl, L. He, Z. Lu and Y. Yin, Adv. Mater., 2009, 21, 4259-4264.

18. R. Xuan and J. Ge, J. Mater. Chem., 2012, 22, $367-$ 372.

19. H. Hu, C. Chen and Q. Chen, J. Mater. Chem. C, 2013, 1, 6013-6030.

20. J. Ge, S. Kwon and Y. Yin, J. Mater. Chem., 2010, 20, 5777-5784.

21. J. Ge, L. He, Y. Hu and Y. Yin, Nanoscale, 2011, 3, 177-183.

22. Y. Zhao, X. Zhao and Z. Gu, Adv. Funct. Mater., 2010, 20, 2970-2988.

23. J. Ge and Y. Yin, Angew. Chem., Int. Ed., 2011, 50, $1492-1522$

24. I. B. Burgess, M. Loncar and J. Aizenberg, J. Mater. Chem. C, 2013, 1, 6075-6086.

25. F. Fleischhaker, A. C. Arsenault, F. C. Peiris, V. Kitaev, I. Manners, R. Zentel and G. A. Ozin, $A d v$. Mater., 2006, 18, 2387-2391.

26. V. L. Alexeev, A. C. Sharma, A. V. Goponenko, S. Das, I. K. Lednev, C. S. Wilcox, D. N. Finegold and S. A. Asher, Anal. Chem., 2003, 75, 2316-2323.

27. V. L. Alexeev, S. Das, D. N. Finegold and S. A. Asher, Clin. Chem., 2004, 50, 2353-2360.

28. S. A. Asher, V. L. Alexeev, A. V. Goponenko, A. C. Sharma, I. K. Lednev, C. S. Wilcox and D. N. Finegold, J. Am. Chem. Soc., 2003, 125, 3322-3329.

29. A. C. Sharma, T. Jana, R. Kesavamoorthy, L. Shi, M. A. Virji, D. N. Finegold and S. A. Asher, J. Am. Chem. Soc., 2004, 126, 2971-2977.

30.Y. Zhao, X. Zhao, B. Tang, W. Xu, J. Li, J. Hu and Z. Gu, Adv. Funct. Mater., 2010, 20, 976-982.

31. Y. Zhao, X. Zhao, J. Hu, M. Xu, W. Zhao, L. Sun, C. Zhu, H. Xu and Z. Gu, Adv. Mater., 2009, 21, 569572.

32. Y. Zhao, X. Zhao, J. Hu, J. Li, W. Xu and Z. Gu, Angew. Chem., Int. Ed., 2009, 48, 7350-7352.

33. H. Wang, Q. Chen, Y. Yu, K. Cheng and Y. Sun, J. Phys. Chem. C, 2011, 115, 11427-11434.

34. J. Ge and Y. Yin, Adv. Mater., 2008, 20, 3485-3491.

35. H. Wang, Y. Yu, Q. Chen and K. Cheng, Dalton T., 2011, 40, 559-563.

36. L. He, Y. Hu, M. Wang and Y. Yin, ACS Nano, 2012, 6, 4196-4202. 